\title{
Multibody Dynamics Simulation of an All-Wheel-Drive Motorcycle for Handling and Energy Efficiency Investigations
}

\author{
J.W. Griffin* and A.A. Popov \\ Faculty of Engineering, University of Nottingham, \\ University Park, Nottingham, NG7 2RD, UK
}

Received 18 June 2014. Accepted 6 February 2017

\begin{abstract}
It is now possible, through electrical, hydraulic or mechanical means, to power the front wheel of a motorcycle. The aim of this is often to improve performance in limit-handling scenarios including off-road low-traction conditions and on-road high-speed cornering. Following on from research into active torque distribution in 4-wheeled vehicles, the possibility exists for efficiency improvements to be realized by reducing the total amount of energy dissipated as slip at the wheel-road contact. This paper presents the results of an investigation into the effect that varying the torque distribution ratio has on the energy consumption of the 2 -wheeled vehicle.

A 13-degree of freedom multibody model was created, which includes the effects of suspension, aerodynamics and gyroscopic bodies. SimMechanics, from the MathWorks@, is used for automatic generation of equations of motion and time-domain simulation, in conjunction with MATLAB and Simulink. A simple driver model is used to control the speed and yaw rate of the motorcycle. The handling characteristics of the motorcycle are quantitatively analysed, and the impact of torque distribution on energy consumption is considered during straight line and cornering situations. The investigation has shown that only a small improvement in efficiency can be made by transferring a portion of the drive torque to the front wheel. Tyre longevity could be improved by reduced slip energy dissipation.
\end{abstract}

Keywords Motorcycle dynamics; multibody modelling; active torque distribution; handling; efficiency.

\section{Introduction}

In conditions of low traction and when high performance is required, there has been a trend towards the use of All-Wheel-Drive (AWD) in vehicles with four or more wheels, as shown in many high performance on-road and off-road vehicles. Not only that, designers have been striving for control over exactly how much torque each of the wheels receives and in which situations. This is demonstrated through the use of locking and limited slip differentials and, more recently, electronically controlled differentials in the form of Active Torque Distribution (ATD) [1]. These systems aim to improve tractive force when one or more wheels has low traction, and to improve handling, stability and safety, especially when the vehicle is approaching the limit of adhesion of its tyres. This trend has not been seen in motorcycles, due to the challenge of applying torque to the front wheel because of the steering system.

There are a few notable exceptions where AWD motorcycles have been developed and tested in the industry; for example, by Öhlins and Christini. Öhlins have developed both off-road and

\footnotetext{
${ }^{*}$ Corresponding author. Email: joseph.griffin@nottingham.ac.uk
} 
on-road motorbikes that use a hydraulic pump and motor to transfer up to $15 \%$ of the torque to the front wheel, depending on the "conditions and throttle position" [2]. Christini have managed to transfer torque to the front wheel of off-road bikes by mechanical means [3]. Here, the front wheel is driven, through a freewheel, at around $80 \%$ of the speed of the rear; thus for torque to be applied, the rear wheel must be spinning faster than the front. Christini claim better hill climbing performance and that, "With the front wheel under power, it is nearly impossible to wash out the front end." Both systems require excess slip at the rear wheel before a drive torque is applied at the front wheel, and the amount of torque transferred is fixed by the design of the system.

Pedal-powered bicycles also exist that have an assistance motor located in the hub of the front wheel. With advances in electric motor technology, it is feasible that electric motors could be used to directly power the front wheel of a motorcycle in a similar fashion, and as such, the amount of torque applied there could be instantly and accurately controlled. However, this may present additional difficulties with the increase in unsprung mass and gyroscopic effects that would need to be investigated.

Results presented by the authors concerning ATD in 4-wheeled vehicles [4] showed that transferring power between the front and rear axles could have an effect on the understeer gradient of the vehicle and could, for example, be used to help prevent a car with an oversteer tendency from spinning during high speed cornering. An investigation into the efficiency of the vehicle showed that a small reduction in energy consumption could be achieved by better distributing the power between the wheels. This is corroborated with results presented in [5]. The difference between front and rear tyre shape and force generating characteristics play a more important role in motorcycle simulation, in comparison to a vehicle with four identical tyres.

Changing the distribution of power between the wheels of a motorcycle could give the designer more control over its handling characteristics. Anecdotal evidence suggests that powering the front wheel makes the motorcycle feel more stable in a turn and gives more traction when accelerating on loose surfaces. This motorcycle model has been developed to investigate the optimal way to distribute torque, and to determine the extent to which the designer can influence the handling, efficiency, and ride quality in a variety of conditions and situations.

The motorcycle model is described in the next section and its validation summarised in Section 3. The handling characteristics of the motorcycle are considered, and the effect of torque distribution on efficiency presented in Section 4, with discussion and conclusions following.

\section{Motorcycle model}

SimMechanics, a graphical multibody modelling suite from The Mathworks [6], was chosen for simulation of the motorcycle, as it allows modelling, control and evaluation of the system in one environment. Equations of motion are generated automatically from a block diagram, based on the specified bodies, joints and constraints. The resulting ODEs are solved using MATLAB solvers, which can be modified to the requirements of the user. MATLAB is used for parameterizing and post-processing.

The motorcycle model was created using a model developed by Meijaard and Popov [7]. Also useful is an example included in the SimMechanics package [8] that uses Sharp's motorcycle model [9] as a basis. A brief overview of the model will be given below.

\section{$2.1 \quad$ Multibody model}

The model consists of seven bodies, each with an associated mass and rotary inertia. There are 13 degrees of freedom specified to describe the relative motion of the bodies. In SimMechanics, the multibody model is described in block diagram form with bodies, joints, sensors and actuators connected in a similar way to Simulink models. Firstly, gravity and an origin in space are defined, then the motorcycle mainframe is connected to the origin with a joint that allows it to move with six degrees of freedom. A Coordinate System (CS) is defined on the body, to which 


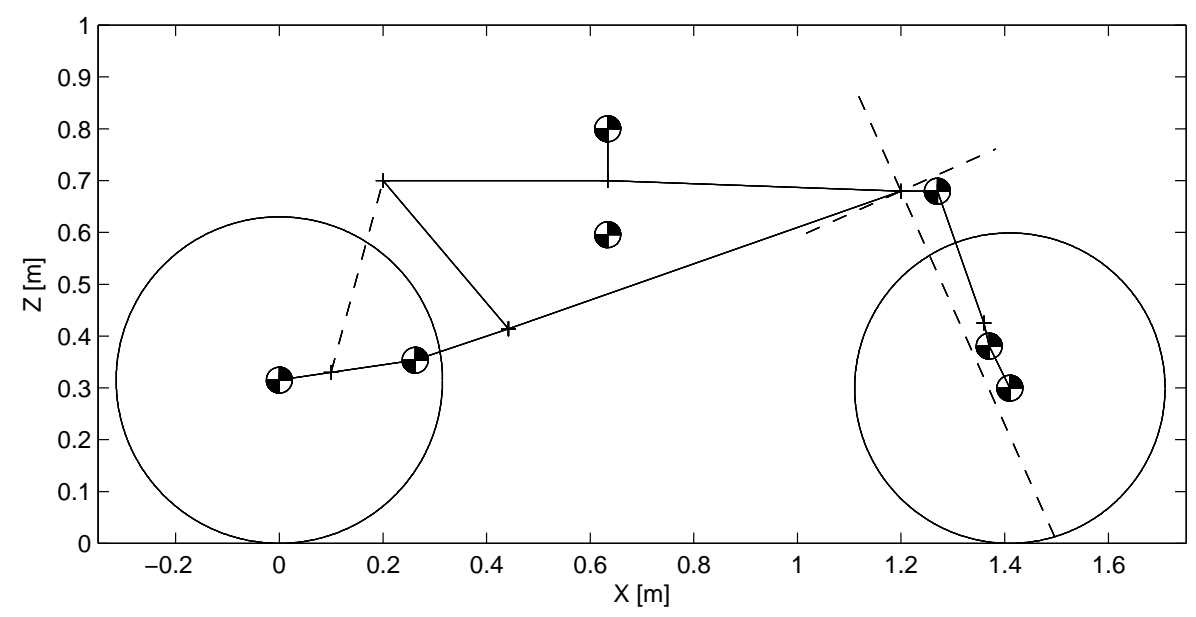

Figure 1: Initial location of the centres of mass and joints of the motorcycle model.

other bodies can be connected through joints, or to which forces and moments can be applied and motions monitored. The rider upper body is connected to a CS at the location of the seat, with a joint that allows the upper body to lean from side to side. The handlebar assembly is connected to the frame with joints that allow steer and twist degrees of freedom at a specified angle to vertical, as shown by dashed lines in Figure 1. Each of the axes have associated spring and damping parameters, and the rider can apply a torque to the handlebars about the steer axis, with an associated reaction on the frame.

At the front, the handlebars are connected, through a prismatic joint incorporating a linear suspension model, to the unsupended forks, and the front wheel is attached to the forks with a revolute joint. Front wheel drive torque is applied to the wheel and has a reaction on lower forks.

At the rear, the swing arm is connected to the main frame with a revolute joint, and a linear spring-damper suspension acts between two specified points of the frame and swing arm, as described in Section 2.3. Finally, the rear wheel is connected with another revolute joint. A drive torque is applied about its axis, with an associated reaction on the mainframe.

The coordinate systems referred to hereafter are described below:

1. the global CS, referred to with a subscript $X Y Z$, which is fixed in space with its origin at an arbitrary point. The $Z$-axis points vertically upwards and the $X-Y$ plane is horizontal and coincident with the ground.

2. the vehicle intermediate $\mathrm{CS}$, with subscript notation $x y z$, which moves with the vehicle and has its origin at the rear wheel contact patch. The $z$-axis remains vertical but the $x$ and $y$-axes are rotated with a yaw angle equal to that of the vehicle's mainframe.

3. the vehicle CS, with subscript notation $x y z v$, which has its origin at the same point as the vehicle intermediate $\mathrm{CS}$ but the $z v$-axis rolls with the motorcycle mainframe about the $x v$-axis and pitches about the $x y$-axis. The $x v-z v$ plane is thus the motorcycle's plane of symmetry.

4. the tyre CS, with subscript notation $x y z t$, which exists for each tyre where each has its origin at the wheel centre. The $z t$-axis remains vertical but the $x t$ - and $y t$-axes are rotated with a yaw angle equal to that of the tyre.

5. the wheel CS, with subscript notation $x y z w$, which also exists for each tyre but here the $z w$-axis cambers with the wheel about the $x t$-axis, which remains horizontal. The $x v-z v$ plane is thus the wheel's plane of symmetry. 


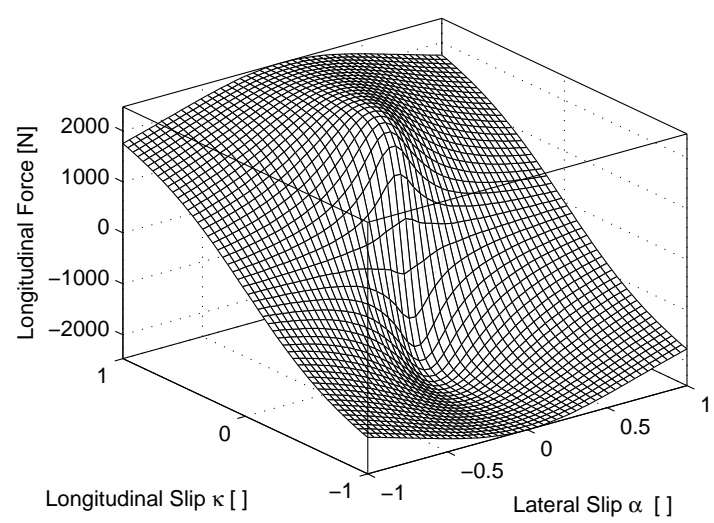

(a) Longitudinal force

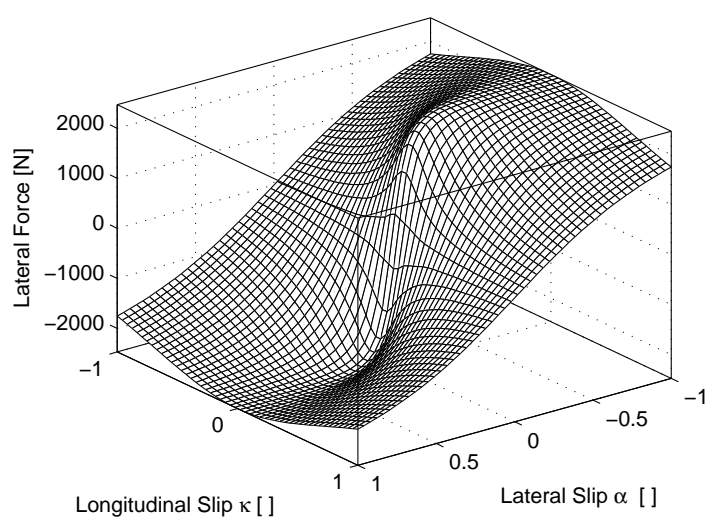

(b) Lateral force

Figure 2: Tyre force plots for combined slip situations, calculated using Meijaard's tyre model [7].

Where subscripts are given as a group, the quantity represents a vector in that coordinate system (e.g. $v_{x y z t}$ is velocity in the tyre CS). Where subscripts are given individually, the quantity refers to the specific component of the vector (e.g. $v_{x t}$ is the velocity in the longitudinal direction of the tyre CS).

\section{$2.2 \quad$ Tyre model}

A tyre model created by Meijaard and Popov [7] is used for the calculation of lateral, longitudinal and vertical forces, along with the aligning moments. The Magic Formula model with motorcycle tyre parameters from [10] was also coded in Simulink and gave similar results. The former was chosen here because it requires fewer parameters and allows faster simulation. Both models include the effects of combined lateral and longitudinal slip and have parameters for typical motorcycle front and rear wheels. Figure 2 shows plots of the lateral and longitudinal tyre forces generated in conditions of combined lateral and longitudinal slip, as calculated with the tyre model. It is clear that when the tyre is generating large forces in one direction, its ability to generate them in the other is diminished. The influence of tyre relaxation length is also included to allow transient manoeuvres to be analysed.

Care has been taken to accurately include the effect of contact patch migration as the wheel cambers, and the influence it has on wheel radius - a characteristic that was not captured by the original SimMechanics model [8] and which was thought to be important to this investigation.

The tyre model requires lateral and longitudinal slip ratios, the vertical deflection and the camber angle, in the tyre CS. The yaw angle is also required to transpose displacements and velocities from the global CS into the tyre CS, and vice versa for the forces and moments. Calculation of the angles is given in Appendix A, while the quantities required by the tyre model, along with the various radii involved, will be described in the next section. Each wheel is calculated separately using values measured at the wheel centre.

\subsubsection{Calculation of slip input quantities}

The longitudinal and lateral slip velocities, $v_{\text {slip } x t}$ and $v_{\text {slip } y t}$ respectively, parallel to the road plane and in the tyre CS, are made non-dimensional using the longitudinal velocity $v_{x t}$ of the wheel centre,

$$
\begin{aligned}
\kappa & =\frac{v_{\text {slip }, x t}}{v_{x t}} \\
\alpha^{\star} & =\frac{v_{y t}}{v_{x t}},
\end{aligned}
$$




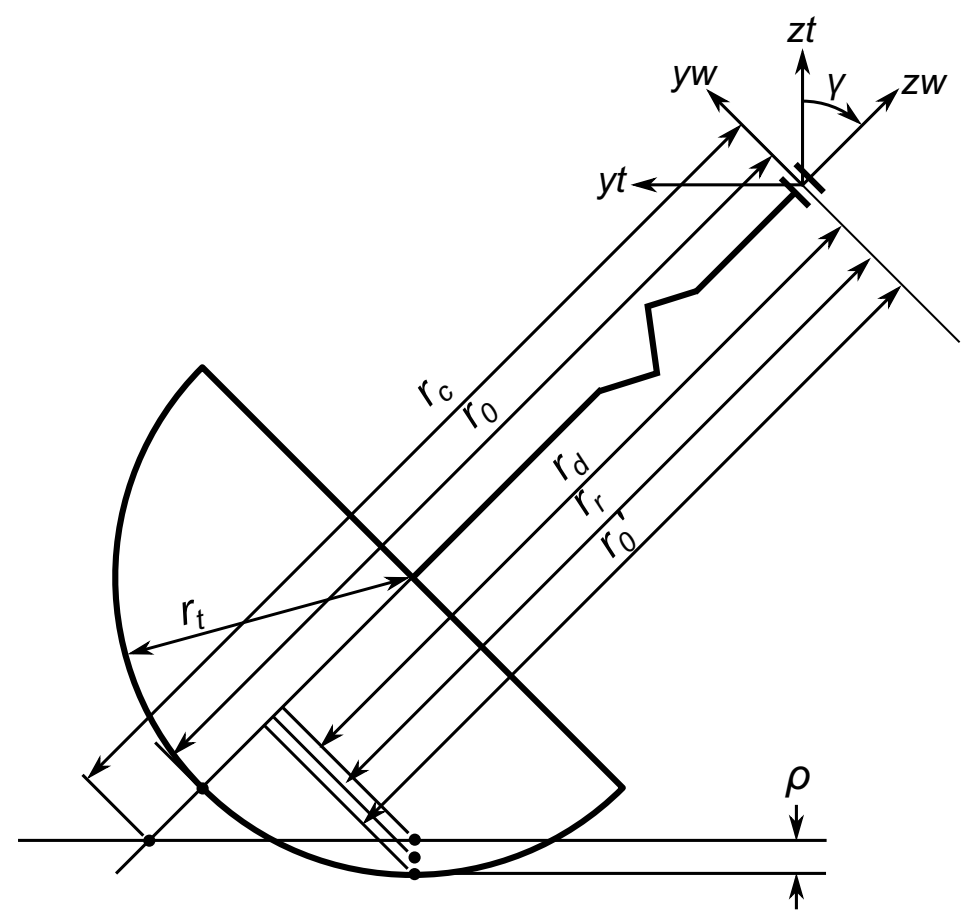

Figure 3: The various radii of a cambered motorcycle wheel

where $\kappa$ is the longitudinal slip ratio, $\alpha^{\star}$ is the lateral slip ratio. The ${ }^{\star}$ differentiates the value from the commonly used slip angle $\alpha=\tan \left(\alpha^{\star}\right)$. The lateral slip velocity is the equal to the lateral velocity of the contact patch, $v_{\text {slip }, y t}=v_{y t}$.

To find the required values in SimMechanics, the vector of velocies at the wheel centre is transformed from the global CS into the yaw-rotated tyre CS with the use of a rotation matrix, as described in Appendix A. The longitudinal slip velocity is calculated using,

$$
\begin{aligned}
v_{s, x t} & =v_{x t}-v_{r} \\
& =v_{x t}-\omega r_{r, z w}
\end{aligned}
$$

in which $v_{r}$ is the rolling velocity and $r_{r, z w}$ is the rolling radius in the wheel CS, shown in Figure 3 and defined in Section 2.2.4. The angular velocity, $\omega$, is taken about the wheel's local $y w$-axis (in the wheel CS).

\subsubsection{Undeformed wheel radius accounting for camber}

To allow calculation of the vertical deflection of the cambered wheel, the radius of the undeformed, yet cambered, wheel $r_{0}^{\prime}$ is required, as shown in Figure 3. The position of the undeformed toroidal tyre surface at the point where it is tangent to a horizontal plane, is calculated from the camber angle and tyre dimensions in the tyre CS as follows:

$$
\boldsymbol{r}_{0, x y z t}^{\prime}=\left[\begin{array}{c}
0 \\
\left(r_{0}-r_{t}\right) \sin \gamma \\
-\left(\left(r_{0}-r_{t}\right) \cos \gamma+r_{t}\right)
\end{array}\right],
$$

where $r_{0}$ is the undeformed radius of the wheel when vertical, and $r_{t}$ is the radius of the tyre toroid. The camber angle $\gamma$ is calculated from the rotation matrix of the wheel, as described in Appendix A. Since the position of the wheel centre is known in the global CS of SimMechanics, the radius vector must be transformed from the wheel CS to the global CS using a rotation matrix that represents a rotation about the global $Z$-axis of the yaw angle $\psi$ of the tyre (see Appendix A). The displacement vectors representing wheel centre location and undeformed 
cambered radius, $s$ and $\boldsymbol{r}_{0}$, respectively, can then be simply added together to find the position of the undeformed wheel in the global CS

$$
\begin{aligned}
& \boldsymbol{r}_{0, X Y Z}^{\prime}=\mathbf{R}_{\psi t} \boldsymbol{r}_{0, x y z t}^{\prime} \\
& \boldsymbol{s}_{0, X Y Z}^{\prime}=\boldsymbol{s}_{\text {wheel centre }, X Y Z}+\boldsymbol{r}_{0, X Y Z}^{\prime}
\end{aligned}
$$

\subsubsection{Deformed wheel radius}

The location of the undeformed tyre described above will be below the centre of the contact patch at the road surface, as shown in Figure 3. The vertical deflection of the tyre $\rho_{z}$, required for the calculation of the normal reaction load, is therefore the $Z$-component of the displacement vector, and the location of the contact patch $s_{C P}$ is found by replacing the $Z$-component with zero.

The positions of the wheel centre and contact patch (on the ground) are now known in the global CS, and thus the distance between them can be calculated. To transform the distance vector to the tyre CS and hense calculate the deformed radius, the inverse of $\mathbf{R}_{\psi t}$ is used

$$
\begin{aligned}
\boldsymbol{r}_{d, X Y Z} & =\boldsymbol{s}_{\text {wheel centre }, X Y Z}-\boldsymbol{s}_{C P, X Y Z} \\
\boldsymbol{r}_{d, x y z t} & =\operatorname{inv}\left(\mathbf{R}_{\psi t}\right) \boldsymbol{r}_{d, X Y Z} .
\end{aligned}
$$

\subsubsection{Wheel rolling radius}

The linear speed of rolling $v_{r}$ is calculated using $v=r \omega$ and, thus, the radius is of critical importance. A definition of rolling radius is chosen such that in free-rolling conditions, the wheel maintains a constant speed without the application of an external torque. Its value will change with vertical load and camber angle, but not with longitudinal slip.

Its value must be between the wheel's undeformed and deformed radii $\left(R_{0, z w}^{\prime}\right.$ and $R_{d, z w}$ in Figure 3, between which the difference is $\rho_{z} \cos (\gamma)$ ). It is smaller than the undeformed radius because compression of the tyre circumference has taken place, but it is larger than the deformed radius because the tyre is more stiff circumferentially than it is radially. As the deformed radius reduces with vertical load, the rolling radius also reduces but at a slower rate.

In the model, using a value from [7], the rolling radius is modelled as being $0.33 \rho_{z}$ below the surface of the road, where $\rho_{z}$ is the vertical distance between the undeformed radius $\boldsymbol{r}_{0}^{\prime}$ and deformed radii $\boldsymbol{r}_{d}$, at that instant.

\subsubsection{Effective wheel radius}

The effective wheel radius is different from the rolling radius and is the (imaginary) point at which the tyre velocity is zero: it is the point about which the tyre appears to rotate at that instant to an observer whose position is fixed in space.

In the case of zero longitudinal slip, the effective radius is equal to the rolling radius, but when slip is present, the effective wheel radius will move up or down, for accelerating and braking, respectively, as shown in Figure 4. This definition means that no force is generated when the wheel is in free-rolling. An alternative definition (with an associated shift in the longitudinal slip vs. force graph) would need a small negative slip speed, and therefore positive force, to maintain a constant speed.

The effective wheel radius can be calculated using

$$
r_{e}=\frac{v_{x t}}{\omega_{y w}}
$$

where $v_{x t}$ is the longitudinal velocity of the tyre and $\omega_{y w}$ is the angular velocity about the local $y w$-axis. Note: this definition differs from some other texts where the effective wheel radius is defined only at zero slip. 


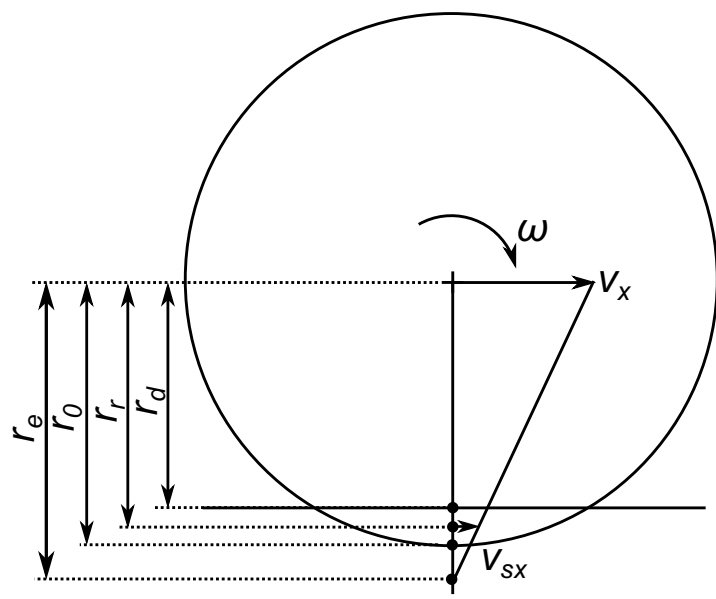

(a) During braking

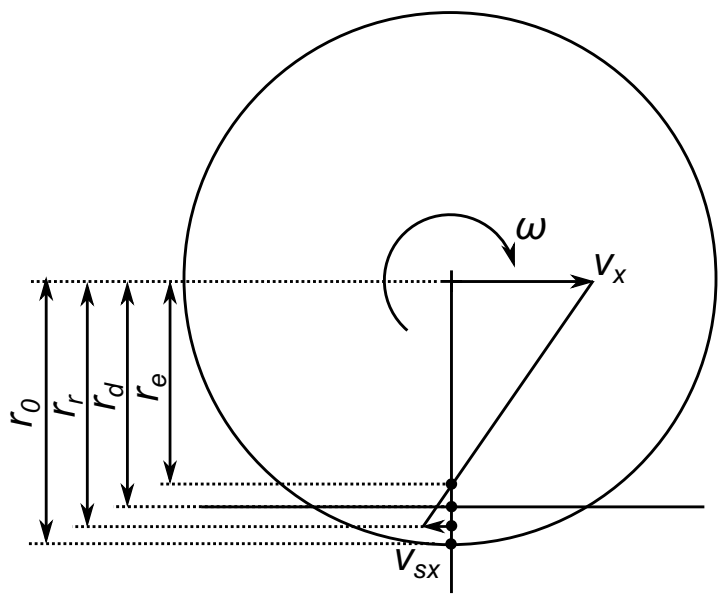

(b) During acceleration

Figure 4: Effective wheel radius variation

\subsubsection{Calculation of the tyre forces and moments}

The tyre forces generated at the contact patch in the tyre CS can be calculated using values of longitudinal slip $\kappa$, lateral slip $\alpha^{\star}$, camber angle $\gamma$ and the veritcal deflection $\rho_{z}$, along with parameters that specify the tyre characteristics taken from [7]. The tyre aligning moment is also calculated about the vertical $z t$-axis. Calculation takes place in a Simulink subsystem with parameters for front and rear tyres specified in MATLAB.

\subsubsection{Application of the tyre forces and moments}

In SimMechanics, forces and moments have to be applied at a CS attached to, and moving with, a body; as such, no suitable CS exists at the contact patch location. Therefore, the forces and moments are first transferred to the wheel centre and then rotated into the global CS before application to the body.

Shifting the application point of the tyre forces means an additional moment, caused by the tyre forces acting at a distance, must be applied. This is calculated using the cross product of the forces and the translation, using the deformed radius $\boldsymbol{r}_{d}$ calculated in Section 2.2.3,

$$
\boldsymbol{M}_{x y z t}^{\prime}=\boldsymbol{M}_{x y z t}+\left(\boldsymbol{r}_{d, x y z t} \times \boldsymbol{F}_{x y z t}\right) .
$$

Both the forces and moments are applied in the global CS, for which the rotation matrix is used again,

$$
\begin{aligned}
\boldsymbol{F}_{X Y Z} & =\mathbf{R}_{\psi t} \boldsymbol{F}_{x y z t} \\
\boldsymbol{M}_{X Y Z} & =\mathbf{R}_{\psi t} \boldsymbol{M}_{x y z t}^{\prime} .
\end{aligned}
$$

\subsection{Suspension forces}

The motorcycle has front and rear suspension, both of which have linear springs and dampers. The front suspension is of the telescopic type, which acts along the line of the front forks. The rear suspension has a linear spring and damper acting between specified points on the main frame and rear swing arm, which results in non-linear rear suspension characteristics. Using the same technique as [8], the following equation is used to find the suspension forces applied at the two connected coordinate systems,

$$
\pm \boldsymbol{F}=\frac{\boldsymbol{s}}{\|\boldsymbol{s}\|}\left[F_{\text {preload }}-\left(\|\boldsymbol{s}\|-s_{\text {nominal }}\right) K-\left(\frac{\boldsymbol{s}}{\|\boldsymbol{s}\|} \times \boldsymbol{v}\right) C\right]
$$


where $\boldsymbol{s}=\boldsymbol{s}_{1}-\boldsymbol{s}_{2}$ is the distance between the two coordinate systems and $\boldsymbol{v}=\boldsymbol{v}_{1}-\boldsymbol{v}_{2}$ is the relative velocity, when the global CS is used. $K$ and $C$ are the spring and damping coefficients, respectively. $\|s\|=\sqrt{s_{X}^{2}+s_{Y}^{2}+s_{Z}^{2}}$, and thus $\frac{s}{\|s\|}$ represents a unit vector in the direction between the two connected points.

\subsection{Aerodynamic forces}

Aerodynamic lift and drag forces are calculated for the current longitudinal vehicle speed, $v_{x v}$ and applied at a point specified on the motorcycle mainframe corresponding to the centre of pressure. The forces are then transformed from the vehicle intermediate CS to the global CS using the rotation matrix of the mainframe,

$$
\begin{aligned}
\boldsymbol{F}_{x y z v} & =\left[\begin{array}{c}
-0.5 \rho_{\mathrm{air}} C_{\mathrm{drag}} v_{x v}^{2} \\
0 \\
-0.5 \rho_{\mathrm{air}} C_{\mathrm{lift}} v_{x v}^{2}
\end{array}\right] \\
\boldsymbol{F}_{X Y Z} & =\mathbf{R}_{v} \boldsymbol{F}_{x y z v} .
\end{aligned}
$$

\subsection{Rider model}

A simple rider model is used that controls two variables independently: the drive torque and the steer torque. The total amount of drive torque is determined using Proportional-Integral (PI) control of the vehicle speed relative to a target. The drive torque is then split between the front and rear wheels according to a ratio specified by the user before simulation,

$$
\begin{aligned}
e_{\text {speed }} & =v_{\text {ref }}-v_{v} \\
\tau_{\text {drive }} & =K_{p} e_{\text {speed }}+K_{i} \int_{0}^{t} e_{\text {speed }} \mathrm{d} t \\
\tau_{\text {rear }} & =T_{r} \tau_{\text {drive }} \\
\tau_{\text {front }} & =\left(1-T_{r}\right) \tau_{\text {drive }}
\end{aligned}
$$

where $v_{v}$ is the resultant vehicle velocity, $\tau$ is drive torque, $T_{r}$ is the rear wheel torque ratio and $K_{p}$ and $K_{i}$ are the proportional and integral gains, set through trial and error at 100 and 20, respectively.

The steering torque is controlled, again using the PI technique, so that the vehicle obtains its target yaw rate. The steer torque $\tau_{\text {steer, }}$, is calculated using

$$
\begin{aligned}
e_{\dot{\psi}} & =\frac{v}{R}-\dot{\psi}_{v} \\
\tau_{\text {steer }} & =-\left(K_{p} e_{\dot{\psi}}+K_{i} \int_{0}^{t} e_{\dot{\psi}} \mathrm{d} t\right),
\end{aligned}
$$

where $R$ is the target radius, which can change with time and can be infinity for straight line driving. $\frac{v}{R}$ is the yaw rate of a vehicle driving in a circle of radius $R$ at velocity $v$. The proportional and integral gains were set through trial and error at 20 and 2, respectively.

In all situations, the motorcycle is released from a vertical position at the origin of the global CS at a specified initial speed. The rider model then attempts to control the vehicle by using the throttle and steer torque inputs to achieve the manoeuvre requested by the user. In the steady state situations presented here, the rider's body is assumed to stay in the plane of the motorcycle.

\subsection{Model outputs}

Data such as slip ratios, and tyre forces and moments, are saved from the tyre models after simulation, along with position and velocity data for each of the bodies. The datasets can then be compared to draw conclusions from the different scenarios. 


\section{Model validation}

To confirm the accuracy of the simulation, various checks are carried out, ensuring that forces and moments balance in steady state, and that power supply and dissipation are equal. The validation process is similar to that used in [11].

\subsection{Force balance}

It is necessary to check that the sum of the tyres forces is equal to the sum of the aerodynamic, inertial and gravitational forces,

$$
\boldsymbol{F}_{\text {error }}=\sum_{i} \boldsymbol{F}_{i \text { tyre }}+\boldsymbol{F}_{\text {aero }}+\sum_{j} m_{j}(\boldsymbol{\omega} \times \boldsymbol{v}+\boldsymbol{g})
$$

for $i=$ front, rear wheels and $j=1, \ldots, 7$ masses. $\boldsymbol{F}_{\text {tyre }}$ is the vector of tyre forces, and $\boldsymbol{F}_{\text {aero }}$ is the vector of aerodynamic lift and drag forces acting on the vehicle. $\sum_{j} m_{j}$ represents the sum of all the machine's constituent masses, $\boldsymbol{v}$ is the velocity vector of the main body and $\boldsymbol{\omega}$ is its angular velocity vector, such that $\boldsymbol{\omega} \times \boldsymbol{v}$ gives the inertial acceleration vector. $\boldsymbol{g}$ is the gravitational acceleration vector, which in this case is $\left[\begin{array}{lll}0 & 0 & -9.81\end{array}\right]$.

Care must be taken that all of the force and velocity vectors are transformed into the same CS for the balance: the vehicle intermediate CS is used here.

\subsection{Moment balance}

The moments applied to the system should also sum to zero in steady state motion. A reference point is chosen and the effect of the forces acting at a distance from this reference point create the moments used in the balance. The reference point can be any point in space but, for simplicity, the centre of mass of the mainframe is chosen here. Again, the vehicle intermediate CS is used:

$$
\begin{aligned}
\boldsymbol{M}_{\text {error }}= & \sum_{i}\left(\boldsymbol{M}_{i \text { tyre forces }}+\boldsymbol{M}_{i \text { tyre moments }}+\boldsymbol{M}_{\text {igyroscopic }}\right) \\
& +\sum_{j}\left(\boldsymbol{M}_{j \text { inertial }}+\boldsymbol{M}_{\text {jgravitational }}\right)+\boldsymbol{M}_{\text {aerodynamic }}
\end{aligned}
$$

for $i=$ front, rear wheels and $j=1, \ldots, 7$ masses. Where the moments are generated by forces acting at a distance from the reference point, as is the case for the tyre forces, and the aerodynamic, inertial and gravitational forces, the moments are calculated using

$$
\boldsymbol{M}=\left(s-s_{\mathrm{ref}}\right) \times \boldsymbol{F},
$$

where $s$ is the position of the body in the global CS. The moments arising from the tyre moments and the gyroscopic moments are summed directly. Since the applied driving torques to the front and rear wheels each have equal and opposite reactions on the forks and mainframe respectively, they are not included in the moment balance.

\subsubsection{Calculation of gyroscopic moments}

The moments generated through gyroscopic precession of the rotating wheels are calculated for each of the wheels using

$$
\boldsymbol{M}=\dot{\boldsymbol{H}}+\boldsymbol{\Omega} \times \boldsymbol{H},
$$

where $\boldsymbol{H}$ is the angular momentum of the gyroscope and $\boldsymbol{\Omega}$ is the angular velocity of the reference frame [12]. In steady state, the rate of change of angular momentum $\dot{\boldsymbol{H}}$ is zero. Here again, one must be careful with the coordinate systems. 
Angular momentum is calculated in the wheel CS using

$$
\boldsymbol{H}^{\prime}=\mathbf{I} \boldsymbol{\omega},
$$

where $\mathbf{I}$ is the $3 \times 3$ inertia matrix of the body and $\boldsymbol{\omega}$ is the angular velocity in the CS mentioned above. The angular momentum is transformed into the tyre CS, which has only a yaw rotation through the use of a rotation matrix for a camber rotation:

$$
\boldsymbol{H}=\mathbf{R}_{\gamma} \boldsymbol{H}^{\prime} .
$$

Gyroscopic moments can now be calculated for each of the rotating bodies in their own yaw-rotated tyre CS, all that remains is to transform them from that into the CS of the chosen reference point, i.e. the vehicle intermediate. This is achieved through the use of a yaw rotation matrix where the yaw angle used is the relative yaw angle between the body and the reference frame

$$
\begin{aligned}
\psi_{\text {rel }} & =\psi-\psi_{\text {ref }} \\
\boldsymbol{M}_{\text {gyro }} & =\mathbf{R}_{\psi_{\text {rel }}}\left(\boldsymbol{\omega}_{v} \times \boldsymbol{H}\right) .
\end{aligned}
$$

\subsection{Power balance}

Finally, the amount of power supplied by the motor(s) can be checked against the dissipaters in the model,

$$
P_{\text {error }}=\sum_{i}\left(P_{i \text { drive }}+P_{i \text { slip }}+P_{i \text { moments }}\right)+P_{\text {aero }},
$$

for $i=$ front, rear wheels.

The amount of drive power provided by the engine and/or motor(s) is calculated at the wheels, neglecting transmission losses, as follows:

$$
P_{\text {drive }}=\tau \omega
$$

where $\tau$ is torque about the wheel's axle, and $\omega$ is the wheel's angular velocity about its axle.

The amount of power consumed as slip at the wheel-road contact can be calculated as

$$
P_{\text {slip }}=F_{x t} v_{s, x t}+F_{y t} v_{y t},
$$

where $F_{x t}$ and $F_{y t}$ are the longitudinal and lateral tyre forces, and $v_{s, x t}$ and $v_{y t}$ are the longitudinal and lateral slip velocities in the tyre CS.

The amount of power consumed through the tyre aligning moment is

$$
P_{\text {moments }}=M_{z t} \omega_{z t},
$$

where $M_{z t}$ is the tyre aligning moment, and $\omega_{z t}$ is its angular velocity about the vertical axis.

And the amount of power consumed overcoming aerodynamics is

$$
P_{\text {aero }}=\sum \boldsymbol{F}_{\text {aero }} \boldsymbol{v}_{x y z v},
$$

where $\boldsymbol{F}_{\text {aero }}$ is the vector of aerodynamics forces and $\boldsymbol{v}_{x y z v}$ is the vector of velocities of the mainframe.

\section{Results}

\subsection{Handling}

To quantitatively investigate the handling of the motorcycle, an approach similar to the calculation of the understeer gradient for 4-wheeled vehicles is employed, as proposed in [13]. By 


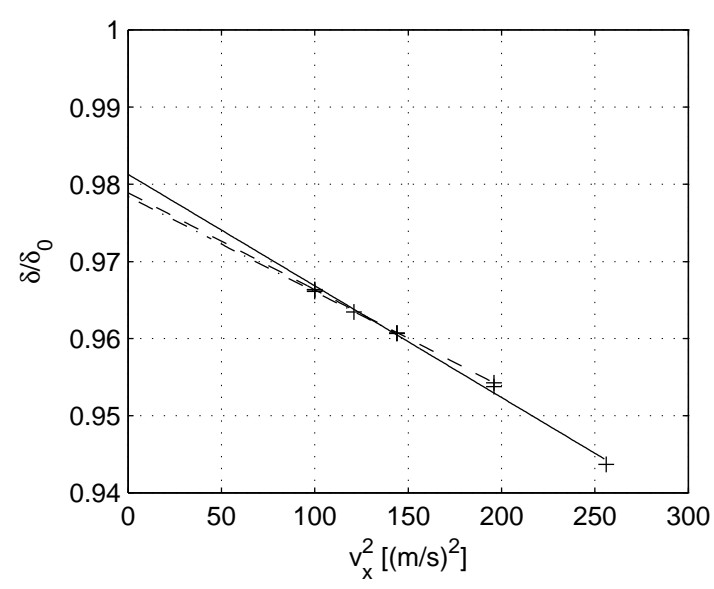

(a) Steer Characteristics

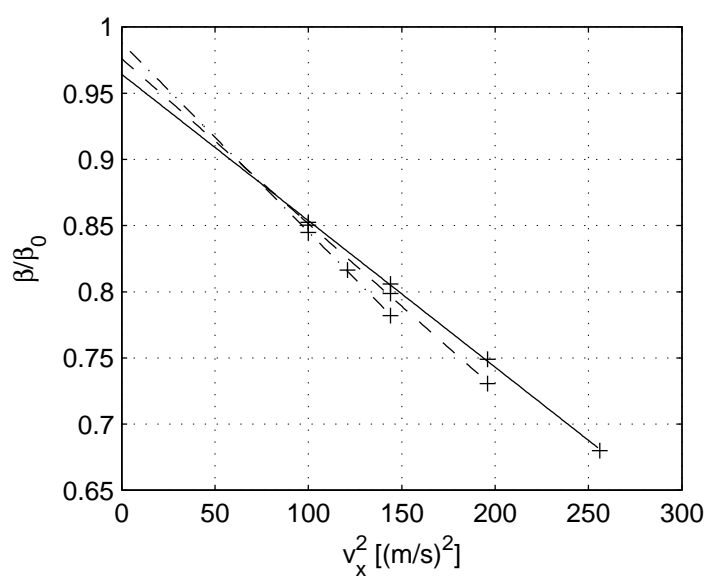

(b) Sideslip Characteristics

Figure 5: Handling characteristics of a 50/50 torque split motorcycle undertaking a constant radius manoeuvre at various speeds. Radius $60 \mathrm{~m}$, dash-dot line; radius $100 \mathrm{~m}$, dashed line; radius $200 \mathrm{~m}$, solid line.

defining $\delta$ as the difference in yaw angle between the front tyre CS and the vehicle intermediate $\mathrm{CS}$, the following equation is found:

$$
\delta-\frac{l}{R}\left(1+K_{\delta} v^{2}\right)
$$

where $l$ is the wheelbase, $R$ is the turning radius, $K_{\delta}$ is the "steer factor" and is similar to the understeer gradient, and $v$ is the velocity. $\delta_{0}$ can be defined as the geometric steer angle, the steer required for to achieve the cornering radius at very low speed, and is equal to $\frac{l}{R}$, thus the equation becomes

$$
\frac{\delta}{\delta_{0}}=1+K_{\delta} v^{2}
$$

Similarly, for the sideslip angle at the centre of mass, the equation is

$$
\beta=\frac{l_{r}}{R}\left(1+K_{\beta} v^{2}\right),
$$

where $l_{r}$ is the distance from the rear wheel to the overall centre of mass. By substituting the geometrically determined sideslip angle at low speed, the equation becomes

$$
\frac{\beta}{\beta_{0}}=1+K_{\delta} v^{2} .
$$

The simulation results for steady state cornering can be plotted to show the relationships described by Equations (36) and (38), as shown in Figure 5.

Figure 5(a) shows that less steer angle is required to negotiate a corner at high speed: the motorcycle exhibits "oversteer" characteristics. A linear motorcycle model with disc-like tyres would produce a plot that passes through zero. Figure 5(b) shows that sideslip angle is reducing, meaning the rear tyre is slipping more than the front, confirming the "oversteer" characteristic.

Since lateral force generating capacity is limited when the wheel is generating high longitudinal forces, the possibility exists for handling and stability improvements to be made by transferring torque to the front wheel.

\subsection{Energy efficiency}

The results for two different steady-state scenarios will be compared to demonstrate the effect of varying the torque distribution between the front and rear wheels: firstly, straight line driving at 
high speed $(45 \mathrm{~m} / \mathrm{s}, 162 \mathrm{~km} / \mathrm{h})$, then secondly, negotiation of a $60 \mathrm{~m}$ radius curve at a moderate speed $(12.5 \mathrm{~m} / \mathrm{s}, 45 \mathrm{~km} / \mathrm{h})$.

The proportion of torque delivered to the rear wheel is varied from $0 \%$ to $100 \%$, with corresponding front wheel torque varying from $100 \%$ to $0 \%$. This assumes an ideal power source with sufficient torque capability at each wheel, and is intended as a basis for the design of a real torque distribution system.

As the values given here are for steady-state situations, the motorcycle was allowed $60 \mathrm{~s}$ simulation time to achieve the desired path and for any transient responses to die away.

\subsubsection{Energy efficiency during straight line driving}

Figure 6(a) shows the total amount of power needed to maintain a speed of $45 \mathrm{~m} / \mathrm{s}$ on a straight and level road with the varying torque distributions. One can see that the total power consumed varies with the amount of power apportioned to the front and rear wheels, with the maximum consumption occurring with purely front wheel drive and the minimum occurring with $70 \%$ of the torque applied at the rear wheel. The minimum occurs with more torque towards the rear, compared to a 50/50 split, because of the differing tyre parameters and loading situations. There is a small reduction in aerodynamic drag as torque is transferred to the rear due to the aerodynamic drag changing with the pitch of the motorcycle.

The absolute magnitude of the change in power consumption is small: even at this relatively high speed, the potential improvement is only around $1.6 \%$, which is insignificant in comparison with other factors in the real world.

Figure 6(b) shows that the longitudinal slip speed of the tyres varies considerably with the torque distribution ratio. Using Equation (32), the amount of power that is dissipated as slip can be calculated. Figure 6(c) shows how much power each wheel dissipates through slip for the various torque distributions. The energy dissipated by the tyres are approximately equal when around torque is divided evenly between the wheel. The plots are not perfectly symmetric because of the different tyre characteristics and loading situations.

These results suggests that motorcycle tyre longevity could be improved by better distributing the torque, meaning that the rear tyre might not have to be replaced as often. This would be especially useful in motorcycles that do a lot of motorway miles when a flat band is often worn on the rear wheel, with an associated negative impact on handling.

\subsubsection{Energy efficiency during steady-state cornering}

The second situation is that of a motorcycle negotiating a constant radius curve, or circle. Figure 7(a) shows that in this situation, the most efficient way to distribute the torque is again to give the rear wheel around $70 \%$ of the torque; the least efficient distribution is with purely front wheel drive. Again, however, the magnitude of the potential improvement is small at around $0.2 \%$.

By considering the slip speeds shown in Figure 7(b) one can see that the amount of longitudinal slip varies with the distribution of torque. Because the speeds are lower, the aerodynamic forces are smaller and the magnitude of the longitudinal slip speed is lower.

Figure $7(\mathrm{c})$ shows the minimum longitudinal slip power consumption for each individual wheel occurs when it is receiving a small amount of power. The minimum power consumption for both wheels together again occurs when power is distributed approximately evenly. Because lateral slip speed, and therefore lateral slip power consumption, is high for all ratios, the variation in longitudinal slip is not as important as in the straight line situation.

\section{Discussion}

The results have shown that only small energy-efficiency improvements are possible in steady state conditions, although this varies with situation. There may well be more improvement possible during acceleration or for hill-climbing manoeuvres, especially on loose ground. 


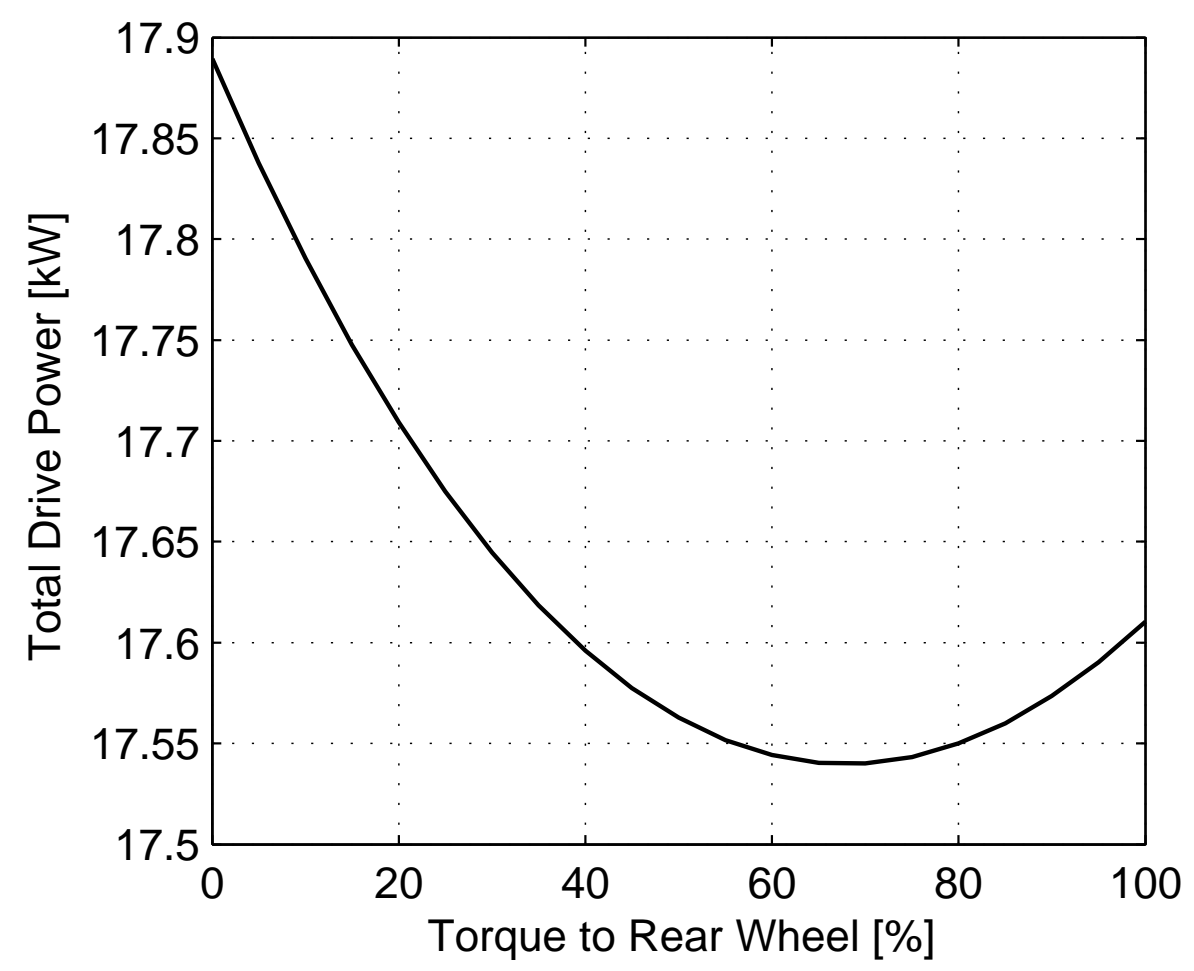

(a) Total power applied

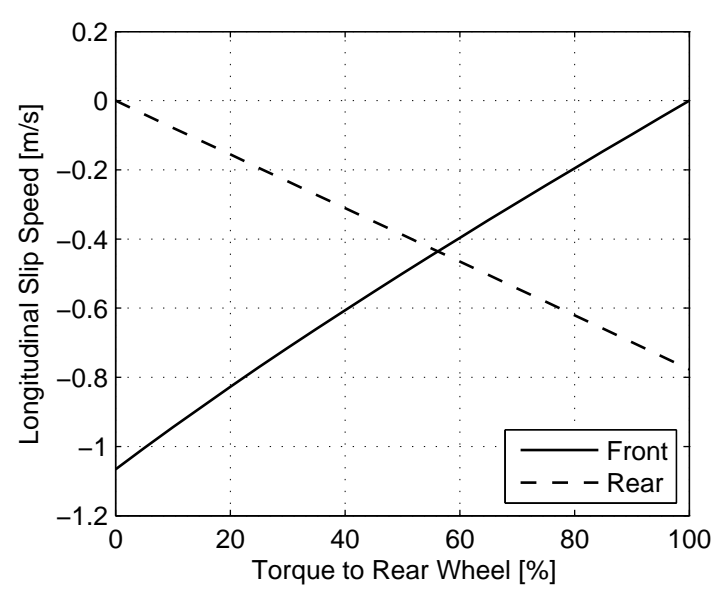

(b) Longitudinal slip speed

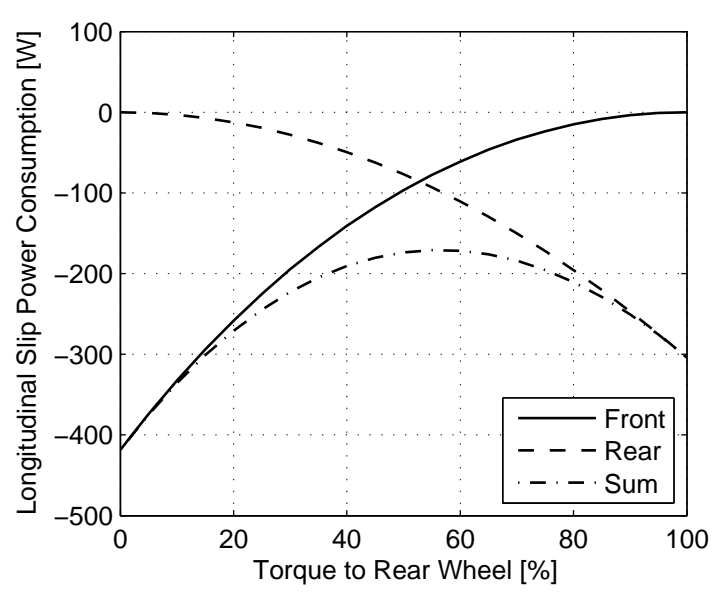

(c) Longitudinal slip power comsumption

Figure 6: Data plots for straight line driving at $45 \mathrm{~m} / \mathrm{s}$ 


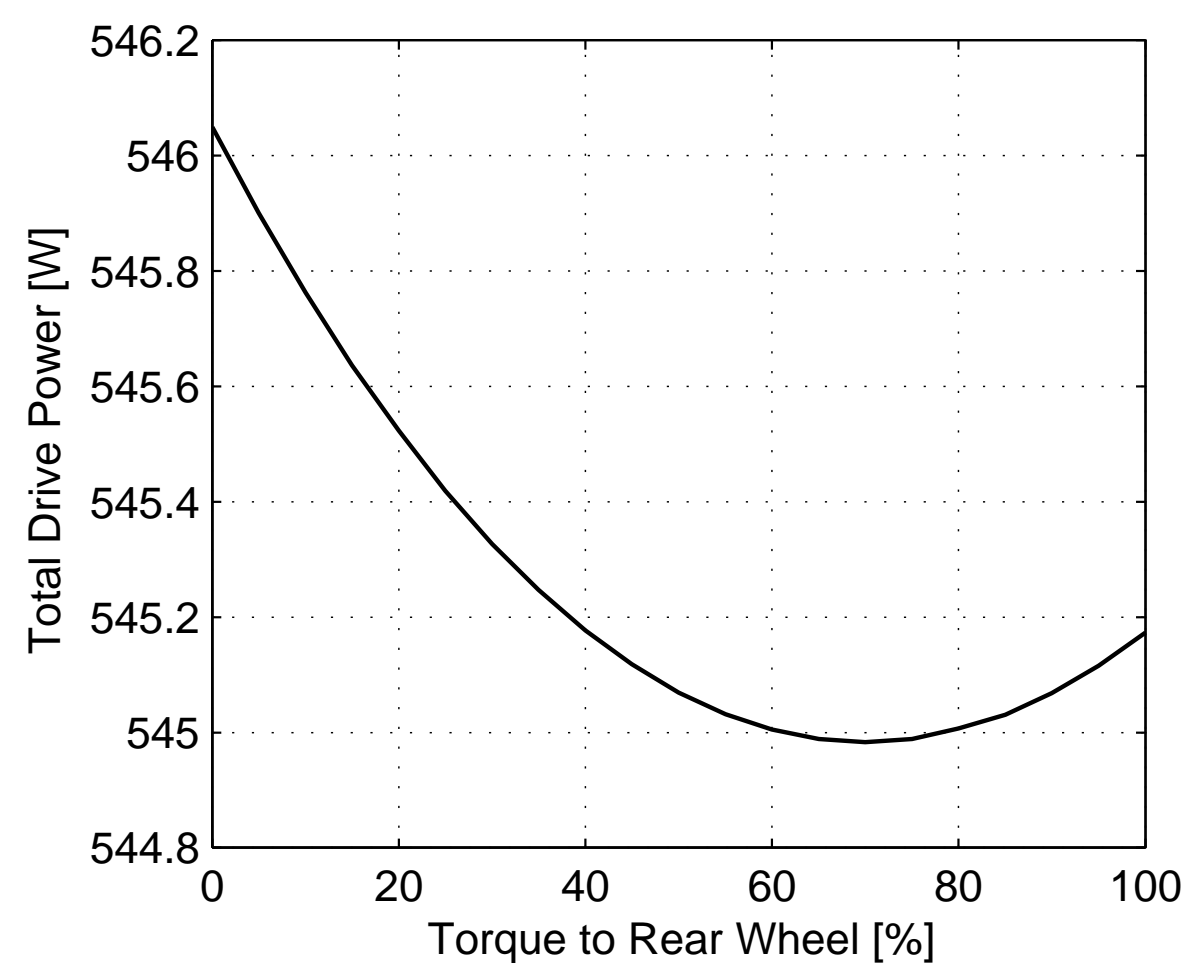

(a) Total power applied

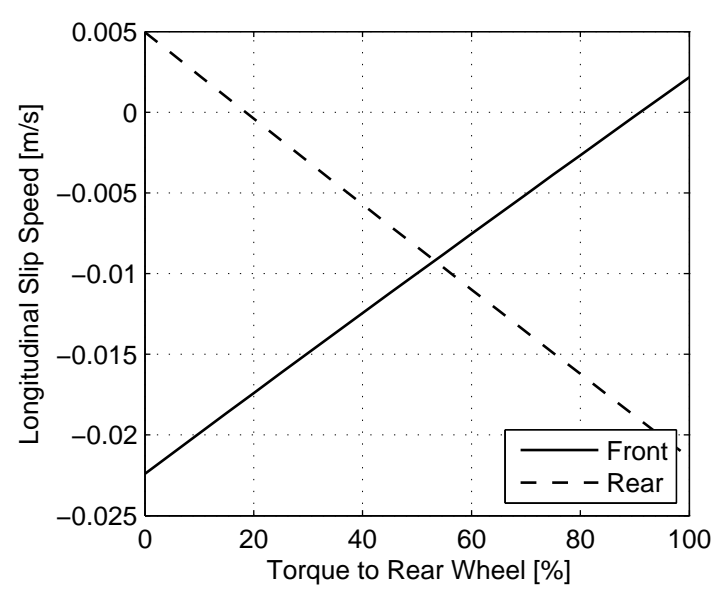

(b) Longitudinal slip speed

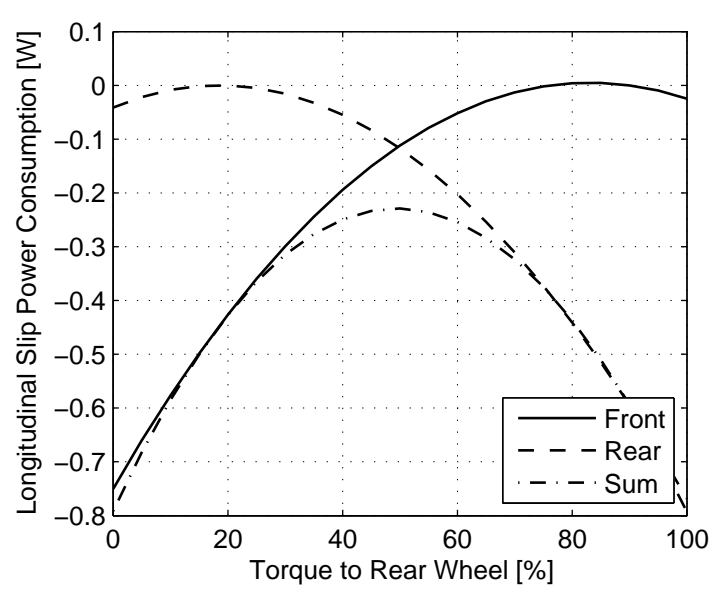

(c) Longitudinal slip power comsumption

Figure 7: Data plots for negotiation of a $60 \mathrm{~m}$ radius curve at $12.5 \mathrm{~m} / \mathrm{s}$ 
The most efficient way to minimise the slip power consumption, and therefore make the tyres last longer, appears to be with the power divided approximately evenly between the tyres. The results depend heavily on tyre characteristics, loading situation and on the environment, with aerodynamic effects dominating the power consumption.

The effect of torque distribution on the range of stability of the motorcycle has, so far, not been investigated. Moreover, the impact on the quality of the ride, in the opinion of a rider, has not been tested and would be difficult to predict.

Currently, powering the front wheel of a motorcycle is difficult and expensive, and such motorcycles are sold at a premium. An electrically driven front wheel opens up the interesting opportunity for energy regeneration under heavy breaking, although there are issues about increasing unsprung mass still to be addressed.

\section{Conclusions}

The presented model has allowed insight into the handling and energy-efficiency of motorcycles with Active Torque Distribution between the front and rear wheels.

ATD in motorcycles has the potential to save only a small amount of energy in the steadystate cases presented here, and associated losses in the necessary drive system may well negate the reduction completely.

ATD does have the potential to reduce the total amount of slip at the wheel-road contacts, especially slip at the rear wheel, which would have an associated increase in tyre longevity, but it seems unlikely that the improvements would be worth the extra cost and complication.

\section{Future work}

Further handling investigations will be undertaken to study the effect of torque distribution on motorcycle handling qualities and stability.

New tyre models will be implemented to allow handling and efficiency investigations on loose or low-friction ground.

It would be feasible to modify the parameters to represent those of a pedal bike to investigate the efficiency of assistance motors and the influence on handling.

\section{Acknowledgement}

This work has been supported by a research grant from the Engineering and Physical Sciences Research Council (EPSRC) of the United Kingdom.

\section{References}

[1] D. Piyabongkarn, J. Lew, R. Rajamani, and J. Grogg, Active Driveline Torque-Management Systems, IEEE Control Systems Magazine 30 (2010), pp. 86-102.

[2] L.J. (Öhlins), 2WD - The Complete Story, (2013), Available at http://www.ohlins.com/Checkpoint-Ohlins/2WD—The-Complete-Story/.

[3] Christini Technologies Inc., The Basics of AWD; Available at http://www.christini.com/awd-technology/about-the-technology.

[4] J. Griffin and A. Popov, Comparisons of Active Vehicle Drivelines and Investigations into their Efficiency, in The 23rd International Symposium on Dynamics of Vehicles on Road and Tracks, Qingdao, China, 2013. 
[5] M. Abe and Y. Kano, A Study on Active Vehicle Chassis Control Reducing Dissipation Energy by Tire Slip for Full Drive-By-Wire Electric Vehcile, in The 23rd International Symposium on Dynamics of Vehicles on Road and Tracks, Qingdao, China, 2013.

[6] T. Mathworks, MathWorks United Kingdom - Products and Services; Available at http://www.mathworks.co.uk/products/.

[7] J. Meijaard and A. Popov, Multi-body modelling and analysis into the non-linear behaviour of modern motorcycles, Proceedings of the Institution of Mechanical Engineers, Part K: Journal of Multi-body Dynamics 221 (2007), pp. 63-76.

[8] G. Wood, Motorcycle: Full Nonlinear Motion; Available at www.mathworks.co.uk/help/physmod/sm/examples_v1/motorcycle-full-nonlinearmotion.html.

[9] R. Sharp and D. Limebeer, A Motorcycle Model for Stability and Control Analysis, Multibody System Dynamics 6 (2001), pp. 123-142.

[10] R. Sharp, S. Evangelou, and D. Limebeer, Improved modelling of motorcycle dynamics, in ECCOMAS Thematic Conference on Advances in Computational Multibody Dynamics, 2003.

[11] S. Evangelou, Control and Stability Analysis of Two-wheeled Road Vehicles, University of London, 2004.

[12] J. Meriam and L.G. Kraige Engineering Mechanics: Dynamics, 7 John Wiley \& Sons, 2012.

[13] I. Kageyama and Y. Kuriyagawa, Study on Influence of Rider's Lean Angle on Two-wheeled Vehicle Behavior, in 10th International Symposium on Advanced Vehicle Control, Loughborough, 2010, pp. 355-360.

\section{A The rotation matrix}

\section{A.1 Definition}

In SimMechanics, the orientation of a body is given by a rotation matrix. The rotation matrix $\mathbf{R}$ is a matrix that transforms a vector from the body-fixed coordinate system (CS) to the global, or space-fixed, CS, as in

$$
r=\mathbf{R} r^{\prime}
$$

with space-fixed coordinates $\boldsymbol{r}$ and body-fixed coordinates $\boldsymbol{r}^{\prime}$ [?]. Its inverse $\operatorname{inv}(\mathbf{R})$ can be used to transform space-fixed coordinates into body-fixed coordinates. In three-dimensional space, it is a $3 \times 3$ matrix,

$$
\mathbf{R}=\left[\begin{array}{lll}
R_{11} & R_{12} & R_{13} \\
R_{21} & R_{22} & R_{23} \\
R_{31} & R_{32} & R_{33}
\end{array}\right],
$$

which, if known, can be solved to find three parameters that characterise the rotation.

\section{A.2 Calculation of the rotation matrix}

Using aeroplane terminology of yaw, roll and pitch, and notation from tyre studies, rotation matrices representing the three individual rotations are given below.

Yaw is an anti-clockwise rotation of $\psi$ about the global $Z$-axis, with a rotation matrix

$$
\mathbf{R}_{\psi}=\left[\begin{array}{ccc}
\cos (\psi) & -\sin (\psi) & 0 \\
\sin (\psi) & \cos (\psi) & 0 \\
0 & 0 & 1
\end{array}\right]
$$


roll, or camber when referring to tyres, is an anti-clockwise rotation of $\gamma$ about the body's $x$-axis, with a rotation matrix

$$
\mathbf{R}_{\gamma}=\left[\begin{array}{ccc}
1 & 0 & 0 \\
0 & \cos (\gamma) & -\sin (\gamma) \\
0 & \sin (\gamma) & \cos (\gamma)
\end{array}\right] ;
$$

and pitch is an anti-clockwise rotation of $\Omega$ about the body's $y$-axis, with a rotation matrix

$$
\mathbf{R}_{\Omega}=\left[\begin{array}{ccc}
\cos (\Omega) & 0 & \sin (\Omega) \\
0 & 1 & 0 \\
-\sin (\Omega) & 0 & \cos (\Omega)
\end{array}\right]
$$

The above rotation matrices can be used to apply the individual tranformations sequentially, or the rotation matrix representing the complete transformation can be used, which is found by multiplying the matrices together:

$$
\mathbf{R}=\mathbf{R}_{\psi} \mathbf{R}_{\gamma} \mathbf{R}_{\Omega}
$$

\section{A.3 Calculation of yaw and camber angles from the rotation matrix}

In the SimMechanics model, the rotation matrix is known, and the individual rotations are required. By simplifying the rotation matrix calculated in Equation (A.6), the following equations are obtained for the yaw, roll and pitch angles, respectively:

$$
\begin{aligned}
\psi & =-\arctan \left(\frac{R_{12}}{R_{22}}\right), \\
\gamma & =\arctan \left(\frac{R_{32}}{\sqrt{R_{31}^{2}+R_{33}^{2}}}\right), \\
\Omega & =-\arctan \left(\frac{R_{31}}{R_{33}}\right) .
\end{aligned}
$$

